\title{
Selectivity Map for Molecular Beam Epitaxy of Advanced III-V Quantum Nanowire Networks
}

\author{
Pavel Aseev,*,†® Alexandra Fursina, ${ }^{\ddagger}$ Frenk Boekhout, ${ }^{\S}$ Filip Krizek, " Joachim E. Sestoft,"
}

Francesco Borsoi, ${ }^{\dagger}$ Sebastian Heedt, ${ }^{\dagger}$ Guanzhong Wang, ${ }^{\dagger}$ Luca Binci, ${ }^{\dagger}$ Sara Martí-Sánchez, ${ }^{\perp}$ Timm Swoboda, ${ }^{\perp}$ René Koops, ${ }^{\S}$ Emanuele Uccelli, ${ }^{\S}$ Jordi Arbiol, ${ }^{\perp, \# \circledast ~ P e t e r ~ K r o g s t r u p, " ~}$ Leo P. Kouwenhoven, ${ }^{*}, \dagger$ and Philippe Caroff* ${ }^{*}+$

${ }^{\dagger}$ QuTech and Kavli Institute of NanoScience, Delft University of Technology, Lorentzweg 1, 2600 GA Delft, The Netherlands ${ }^{\ddagger}$ Microsoft Station Q at Delft University of Technology, 2600 GA Delft, Netherlands

${ }^{\S}$ QuTech and Netherlands Organization for Applied Scientific Research (TNO), Stieltjesweg 1, 2628 CK Delft, The Netherlands

"Center For Quantum Devices and Station Q Copenhagen, Niels Bohr Institute, University of Copenhagen, 2100 Copenhagen, Denmark

${ }^{\perp}$ Catalan Institute of Nanoscience and Nanotechnology (ICN2), CSIC and BIST, Campus UAB, 08193 Bellaterra, Barcelona, Catalonia, Spain

\#ICREA, Passeig de Lluís Companys 23, 08010 Barcelona, Catalonia, Spain

\section{Supporting Information}

ABSTRACT: Selective-area growth is a promising technique for enabling of the fabrication of the scalable III-V nanowire networks required to test proposals for Majorana-based quantum computing devices. However, the contours of the growth parameter window resulting in selective growth remain undefined. Herein, we present a set of experimental techniques that unambiguously establish the parameter space window resulting in selective III-V nanowire networks growth by molecular beam epitaxy. Selectivity maps are constructed for both GaAs and InAs compounds based on in situ characterization of growth kinetics on $\mathrm{GaAs}(001)$ substrates, where the difference in group III adatom desorption rates between the III-V surface and the amorphous mask area is identified as the primary mechanism governing selectivity. The broad applicability of this method is demonstrated by the successful realization of high-quality InAs and GaAs nanowire networks on GaAs, InP, and InAs substrates of both (001) and (111)B orientations as well as homoepitaxial InSb nanowire networks. Finally, phase coherence in Aharonov-Bohm ring experiments validates the potential of these crystals for nanoelectronics and quantum transport applications. This work should enable faster and better nanoscale crystal engineering over a range of compound semiconductors for improved device performance.

KEYWORDS: Selective-area growth, epitaxy, selectivity, III-V nanowire; InAs, GaAs, molecular beam epitaxy
T nterest in hybrid superconductor/semiconductor nanowires (NWs) is rising rapidly thanks to their promises to host Majorana zero modes. These pseudoparticles could serve as a basis for fault-tolerant quantum computing with exceptionally long coherence times of individual qubits, revolutionizing the way we are doing computations. ${ }^{1-3}$ Most of the advances in Majorana-related transport experiments are demonstrated in single III-V semiconductor NWs covered with an s-wave superconductor, ${ }^{4-11}$ where NWs are grown by employing the vapor-liquid-solid (VLS) method. ${ }^{12,13}$ However, the VLSgrown NWs can form networks with only a limited number of interconnections ${ }^{14-18}$ and are inherently non-scalable because of the requirement for post-growth nanomanipulation of the individual nanostructures onto a host substrate to allow for the device-processing steps. These limitations must be overcome to pave the way toward Majorana-based integrated circuits.

A natural alternative to the VLS approach is the selective-area growth (SAG) technique in conjunction with a change of geometry from free-standing vertical nanowires to an in-plane geometry of nanowire networks. SAG proceeds similarly to planar 2D growth but only inside lithographically defined openings in an amorphous mask on a crystalline substrate without any catalysts. This approach allows the controlled formation of scalable in-plane NW networks in which the shape

Received: September 14, 2018

Revised: November 15, 2018

Published: December 6, 2018 


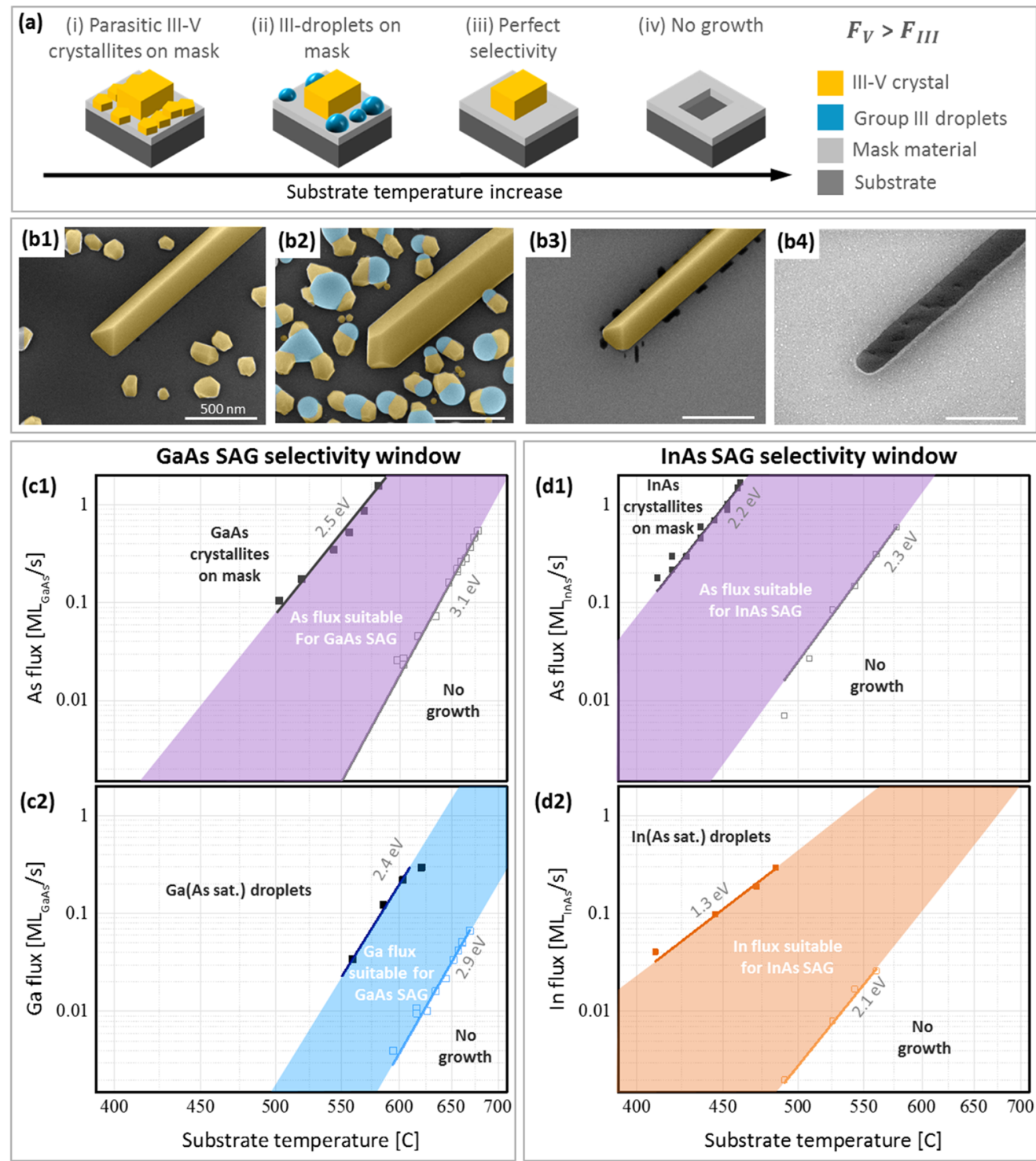

Figure 1. Selectivity maps for GaAs and InAs SAG. (a) Illustration of the III-V growth modes on patterned substrate at different substrate temperatures for fixed $F_{\mathrm{V}}$-to- $F_{\mathrm{III}}$ flux ratio $\left(F_{\mathrm{V}}>F_{\mathrm{III}}\right)$. False-color SEM images of growth modes in the case of homoepitaxial GaAs SAG $\left(F_{\mathrm{Ga}}=0.26\right.$ $\mathrm{ML}_{\mathrm{GaAs}} / \mathrm{s}$ and $F_{\mathrm{As}}=0.96 \mathrm{ML}_{\mathrm{GaAs}} / \mathrm{s}$ ) grown at (b1) $610^{\circ} \mathrm{C}$ with post-growth cooling under As flux leading to GaAs crystallite formation (yellow) on the mask, (b2) $600^{\circ} \mathrm{C}$ with post-growth cooling under gradually decreasing As flux leading to Ga (As-saturated) droplet (blue) accumulation on the mask and partially converted into Ga/GaAs clusters (yellow) upon cooling, (b3) $630{ }^{\circ} \mathrm{C}$ leading to selective growth, and (b4) $690{ }^{\circ} \mathrm{C}$ leading to GaAs evaporation during the growth inside the openings. Scale bars are $500 \mathrm{~nm}$. Selectivity maps for GaAs and InAs on GaAs(001) substrate: (c1, d1) upper and lower bounds of group V elemental flux suitable for SAG corresponding to its desorption from the mask with partial coverage by group III element clusters (filled points) and from III-V surface (empty points), respectively; (c2, d2) upper and lower bounds of group III elemental flux suitable for SAG corresponding to its desorption from the mask surface (filled points) and from III-V surface (empty points). Effective activation energies for each line are marked next to it.

complexity and control over NW dimensions is obtained by design.

While SAG is a mature technique regularly used for epitaxial lateral overgrowth (ELOG) for fin field-effect transistors $(\text { FinFET })^{19}$ and III-V/Si integration, ${ }^{20}$ only recently studies have highlighted its potential for low-temperature transport physics. ${ }^{21-26}$ Among available SAG compatible synthesis techniques, molecular beam epitaxy (MBE) provides the key advantage of allowing both in situ high-quality superconductor deposition (e.g., epitaxial Al) proven to be crucial for Majoranabased devices ${ }^{8}$ and the lowest levels of impurity incorporation thanks to the lack of chemical precursors and ultrahigh-vacuum conditions.
Using the SAG technique for III-V NWs allows their location and in-plane geometry to be lithographically defined prior to growth by openings in an amorphous mask covering a crystalline substrate. ${ }^{27}$ Depending on growth conditions, four possible growth modes can be observed during MBE, as schematically depicted in Figure 1a, and experimentally demonstrated for GaAs SAG, as shown in Figure $1 \mathrm{~b}$ :

(i) nucleation of randomly oriented III-V crystallites on the mask along with single-crystalline III-V growth inside the openings,

(ii) formation of liquid and partially crystallized III/III-V clusters on the mask along with single-crystalline III-V growth inside the openings, 
(iii) selective single-crystalline III-V growth inside the openings, and

(iv) no growth on the mask or inside the openings (III-V decomposition regime).

Despite the initial work on SAG by MBE being reported as early as in the $1970 \mathrm{~s},{ }^{28}$ there is still limited understanding of which processes govern the selectivity. Okamoto and Ohata studied the upper boundary of the selectivity window of $\mathrm{GaAs}^{29}$ and $\operatorname{InAs}{ }^{30} \mathrm{SAG}$ on $\mathrm{GaAs}(001)$ substrate with a $\mathrm{SiO}_{x}$ mask by reflection high-energy electron diffractometry (RHEED). The sticking coefficient of $\mathrm{Ga}$ adatoms on $\mathrm{SiO}_{x}$ as a function of substrate temperature was studied by ex situ scanning electron microscopy (SEM) by several groups. ${ }^{31}-35$ Allegretti et al. ${ }^{36}$ studied the selectivity of GaAs on GaAs(001) substrates with $\mathrm{SiO}_{x}$ mask by in situ SEM and RHEED. The authors measured the critical impinging Ga flux leading to parasitic growth as a function of substrate temperature under constant As flux. Yokoyama et al. ${ }^{37}$ studied the Ga desorption rate from $\mathrm{SiO}_{x}$ as a function of substrate temperature and As flux, but no explanation for the formation of GaAs crystallites on the mask was given. For other III-V compounds, even less information is available. ${ }^{38,39}$ We emphasize that all above-mentioned reports do not distinguish between growth modes $i$ and ii, which, as we show here, is crucial for understanding the fundamental selectivity mechanisms. Therefore, to the best of our knowledge none of the reported studies provide a comprehensive description of the selectivity mechanisms in III-V SAG.

Here, we develop a general set of experimental procedures, socalled selectivity mapping, to unambiguously establish the parameter space (selectivity window) where III-V NW growth proceeds selectively at locations defined by lithography patterning (growth mode iii). To do so, we investigate different SAG modes outlined in Figure 1a and the transitions between them. Selectivity mapping is performed for GaAs and InAs on GaAs $(001)$ substrate with a $\mathrm{SiO}_{x}$ mask surface, and results are extended to the InAs, InP, and GaAs substrates of both (001) and (111)B orientations. Particular interest is paid to the semiinsulating (111)B substrate on which we demonstrate waferscale in-plane InAs NW networks with unprecedented network complexity and a high yield of crossed junctions. Transmission electron microscopy reveals high compositional uniformity and structural quality of both individual NWs and their networks. We probe the quantum-transport properties of selected network geometries such as Aharonov-Bohm interference in loopshaped structures demonstrating quantum coherence, which is crucial for Majorana-based quantum computational applications. ${ }^{14,40} \mathrm{We}$ emphasize that obtained selectivity maps potentially allow the precise growth optimization of III-V SAG without losing selectivity. This is crucial to meet the tight requirements of novel quantum devices.

In this work, we define the term "selectivity" as a set of growth conditions during SAG at which the desorption of group III and $\mathrm{V}$ adatoms from the amorphous mask is high enough to exponentially reduce the chance of parasitic nucleation of III-V crystallites and metal droplet accumulation on the mask and, at the same time, the desorption from the mask openings is low enough to allow III-V growth. The upper bound for impinging group III and V fluxes $\left(F_{\text {III }}\right.$ and $\left.F_{\mathrm{V}}\right)$ suitable for $S A G$ is defined by adatom desorption rate from the mask material. The lower bound is then set by desorption from III-V surface within the mask openings. In general, the desorption rate $\left(F_{A \mid S}^{\text {des }}\right)$ of an element $A$ from a surface $S$ can be described by a simple Arrhenius equation:

$$
F_{A \mid S}^{\mathrm{des}}=R \exp \left(-\frac{E_{\mathrm{D}}(A, S)}{k T}\right)
$$

where $R$ is a frequency factor assumed to be constant over the studied temperature range, $k$ is the Boltzmann constant, $T$ is the surface temperature, and $E_{\mathrm{D}}(A, S)$ is an effective activation energy of desorption that depends on both desorbing species $A$ and the surface properties of $S$. The desorption rate depends linearly on the impinging flux until it reaches a saturation value defined by the eq $1{ }^{41}$

Complete mapping of the selectivity windows for GaAs and InAs SAG on GaAs(001) substrate with a $\mathrm{SiO}_{x}$ mask surface is presented in this work. All samples are grown by MBE, with substrate temperature measured by calibrated pyrometer and impinging and desorption fluxes presented in equivalent monolayers per second $\left(\mathrm{ML}_{\mathrm{III}-\mathrm{V}} / \mathrm{s}\right)$, as discussed in Supporting Information SA. Substrate and mask preparation procedures are described in Supporting Information SB. In situ RHEED measurements are used to construct the selectivity maps with details discussed for GaAs SAG. The same arguments are valid for the InAs SAG as well as for different choice of substrate materials and orientations.

Figure $1 \mathrm{c} 1$ presents the upper and lower bounds of the impinging As flux suitable for GaAs SAG. The upper As bound is measured by monitoring the transition of the RHEED pattern from a halo (corresponding to an amorphous mask surface) to a polycrystalline pattern (indicating formation of randomly oriented III-V crystallites on the mask). ${ }^{31,42}$ This transition is induced by gradually increasing the As flux after a short fixed predeposition of $\mathrm{Ga}$ (equivalent to $5 \mathrm{ML}_{\mathrm{GaAs}}$ ). The transition is rather abrupt, as it only takes tens of seconds to occur when the As flux is higher than the upper As selectivity bound by at least $0.1 \mathrm{ML} / \mathrm{s}$ (Figure 1c1). In contrast, if the impinging As flux is lower than that bound, then no formation of a polycrystalline pattern is observed even after prolonged exposure (e.g., $8 \mathrm{~min}$ at $T=585^{\circ} \mathrm{C}$ and $F_{\mathrm{As}}=0.98 \mathrm{ML}_{\mathrm{GaAs}} / \mathrm{s}$ ). The lower As bound is measured by monitoring of the RHEED reconstruction transition from $(2 \times 4)$ to $(4 \times 2)$, at which the As flux is equal to the impinging Ga flux. ${ }^{43}$ The exponential increase in As flux necessary to maintain $(2 \times 4)$ reconstructions at elevated temperatures is attributed to an increase in As desorption from the III-V surface (Figure 1c1, empty dots). ${ }^{43}$

Figure $1 \mathrm{c} 2$ presents the upper and lower bounds of the impinging Ga flux suitable for GaAs SAG. The upper Ga bound is measured by monitoring the temperature dependence of the RHEED intensity drop during predeposition of a fixed amount of Ga at a fixed As flux. The RHEED intensity drop is calibrated to an amount of deposited Ga at relatively low substrate temperatures $\left(520{ }^{\circ} \mathrm{C}\right)$ where the desorption of $\mathrm{Ga}$ is negligible. ${ }^{43}$ The exponential decrease of the amount of deposited $\mathrm{Ga}$ at elevated temperatures is attributed to suppressed nucleation of $\mathrm{Ga}$ clusters on the mask. If the impinging Ga flux exceeds that bound, while the As flux is kept below the upper SAG bound (Figure 1c1) then a liquid Ga phase accumulates on the mask. This can be confirmed by ex situ SEM (Figure 1b2) with the important requirement that during the sample cooling to room temperature the As flux is adjusted to be below the upper SAG bound at all times. In contrast, if the As flux is maintained, then upon cooling it converts the liquid Ga (As-saturated) phase into GaAs crystallites, which can be 

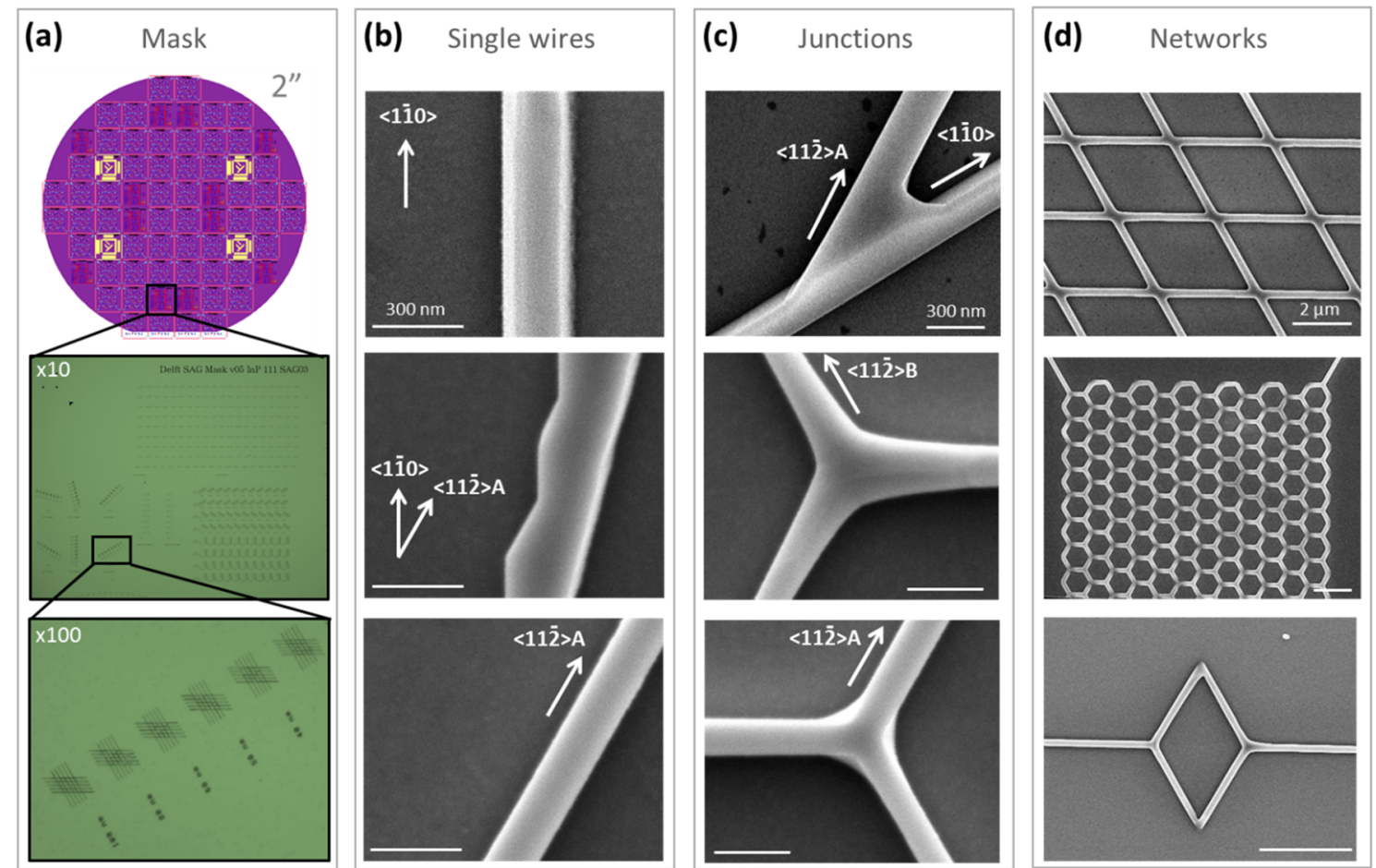

Figure 2. In-plane InAs SAG network morphology on $\operatorname{InP}(111) B$ substrates controlled by high-symmetry crystallographic directions. (a) Design of the mask pattern on a $2 \mathrm{in}$. InP(111)B wafer (top) with 70 unit cells of $5 \mathrm{~mm} \times 5 \mathrm{~mm}$ dimensions (middle) containing various arrays of NW networks (bottom) imaged by optical microscopy after the growth. (b) SEM micrographs displaying uniform faceting of single NWs oriented along the highsymmetry $\langle 1 \overline{1} 0\rangle$ (top) and $\langle 11 \overline{2}>A$ (bottom) directions and non-uniform faceting in the intermediate case (middle). (c) Junctions consisting of different NW families: asymmetrical junction of $\langle 11 \overline{2}>\mathrm{A}$ and $\langle 1 \overline{1} 0\rangle$ (top), symmetrical $\langle 11 \overline{2}>\mathrm{B}$ (middle), and $<11 \overline{2}>\mathrm{A}$ (bottom) with the latter one being more uniform. (d) Example of complex NW networks composed of the $<11 \overline{2}>$ NW family: comb pattern (top), honeycomb pattern (middle), and Aharonov-Bohm ring shape (bottom).

detected by both observation of polycrystalline ring RHEED pattern (refer to Supporting Information SC.3) and ex situ SEM (Figure 1b1). The lower Ga bound is measured by monitoring the onset of the GaAs growth rate at elevated temperatures by standard RHEED oscillations method. ${ }^{32,43,44}$ The obtained data is in perfect agreement with literature reports within a $10{ }^{\circ} \mathrm{C}$ accuracy margin. ${ }^{43}$ If the impinging Ga flux during the growth is lower than the desorption flux then the NW and substrate decomposition occurs instead of growth of III-V as shown in Figure $1 \mathrm{~b} 4 .{ }^{45}$ This effect is not always detrimental, as it can be used for controllable thinning of the III-V nanostructures. ${ }^{46}$

Note that Ga desorption from liquid Ga on the mask is lower than from uncovered mask surface (see Supporting Information SC.6). This suggests that if the impinging Ga flux is higher than the desorption rate from the mask at a chosen substrate temperature, then the Ga droplets accumulate on the mask surface and a substantially higher substrate temperature is needed to desorb it. This is related to different kinetic barriers for evaporation of group III adatoms from the amorphous mask versus from liquid group III droplets. ${ }^{47}$

A detailed description of the techniques and methodology collectively used to obtain the maps in Figure 1 is provided in the Supporting Information SC. The $\mathrm{SiO}_{x}$ mask surface was repeatedly exposed to In and $\mathrm{Ga}$ adatoms during the selectivity-mapping experiments. We have performed atomic force microscopy (AFM) imaging of its surface in areas directly exposed and shielded from group III impinging fluxes (refer to Supporting Information SC.7). No significant mask surface deterioration was revealed, which confirms its robustness against reaction with group III adatoms and ensures the reproducibility of obtained results.

Importantly, we have identified a primary mechanism governing selectivity to be the difference in the group III adatom desorption rates from the amorphous mask versus from III-V crystal. The role of group V desorption rate is considered to be secondary because at any chosen substrate temperature its value is higher than the group III desorption from the same surface. This also suggests that, for commonly used growth conditions during III-V growth (V-to-III ratio of $1-10$ and growth rate of $0.1-0.5 \mathrm{ML} / \mathrm{s})$, the formation of III-V crystallites on the mask (controlled by group $\mathrm{V}$ desorption) is not the main factor limiting the selectivity but, rather, accumulation of group III adatoms in the form of liquid droplets (controlled by group III desorption). Moreover, our results explain well the correlation that samples grown at lower temperatures and cooled under constant group $\mathrm{V}$ flux demonstrate exponentially higher density of III-V crystallites, previously reported for selectivity characterization obtained from ex situ SEM analysis. ${ }^{34,35}$ Indeed, this is because of exponential increase of the accumulation of liquid III droplets at low substrate temperatures, which then get converted into III-V crystallites upon cooling under group V flux. Similar behavior was reported for self-catalyzed VLS nanowires. ${ }^{48,49}$ Therefore, we emphasize the importance of distinguishing between formation of III-V crystallites and liquid III droplets on the mask for correct interpretation of experimental results.

The fundamental understanding gained here allows us to propose that the mechanism of selectivity enhancement by using metal modulated epitaxy $(\mathrm{MME})^{37}$ and migration enhanced 

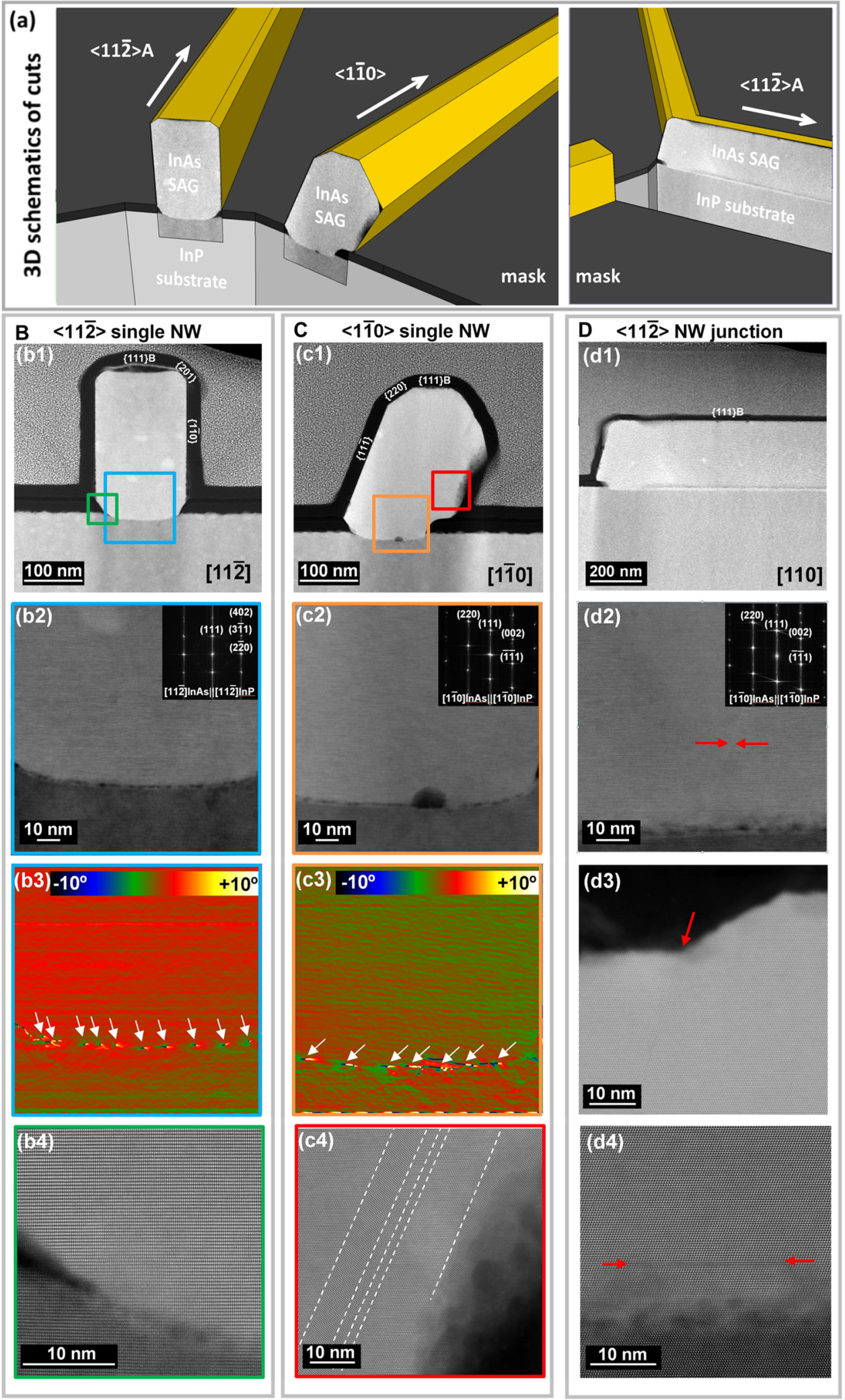

Figure 3. Structural properties of in-plane InAs SAG NWs on $\operatorname{InP}(111) B$ substrates. (a) Schematics of the NW cross-sections under investigation: (left) perpendicular and (right) parallel to the NW in-plane direction. Panel B: cross-sectional STEM analysis of a $<11 \overline{2}>$ nanowire observed through $[1 \overline{2} 1]$ zone axis. (b1) Low-magnification HAADF-STEM micrograph showing an overview of the cross-section and faceting. (b2) Atomic-resolution

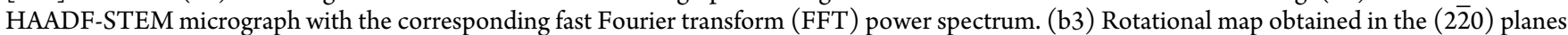
highlighting the presence of periodical misfit dislocations at the InAs-InP interface (white arrows). (b4) Details on the atomic arrangement near the interface with the substrate and mask. Panel C: cross-sectional STEM analysis of a $<1 \overline{1} 0>$-oriented nanowire observed through $[1 \overline{1} 0]$ zone axis. (c1) Low-magnification HAADF-STEM micrograph of cross-sectional cut. (c2) Atomic-resolution HAADF-STEM image with the corresponding FFT and plane identification on the sample interface. (c3) Rotational map applied to the $(\overline{111})$ planes highlighting the presence of the misfit dislocations at the 
Figure 3. continued

InAs/InP interface (white arrows) and (c4) HAADF-STEM image on the overgrown region at the right showing the presence of twin boundaries (white dashed lines). Panel D: Longitudinal cross-sectional HAADF STEM of a $<11 \overline{2}>$-oriented NW junction observed through [1 $\overline{1} 0]$ zone axis. $(\mathrm{d} 1)$ Low-magnification overview of the cut. (d2) Interface with a propagating stacking fault (red arrows). (d3) Stacking fault creating the step on top of the $\mathrm{NW}$ and (d4) in-plane stacking fault originating just above the InAs-InP interface.

epitaxy $(\mathrm{MEE})^{50,51}$ can be clearly attributed to desorption of liquid group III droplets during periodic interruption of group III flux rather than to decomposition and sublimation of III-V crystallites from the mask. ${ }^{52}$ The same explanation is applicable to $\mathrm{H}$-assisted MBE, where hydrogen plasma is used to improve selectivity by forming volatile compounds with parasitic group III droplets ${ }^{42,53}$ and not with III-V crystallites. This explanation agrees well with the reports demonstrating absence of hydrogen promoted decomposition of $\mathrm{GaAs}(001)$ surfaces. ${ }^{53}$

The impinging flux boundaries presented in Figure $1 \mathrm{c} 1, \mathrm{c} 2, \mathrm{~d} 1, \mathrm{~d} 2$ combined present complete selectivity maps for SAG of InAs and GaAs on GaAs(001) substrates with the patterned $\mathrm{SiO}_{x}$ mask surface. Obtained maps detail the boundaries between four growth modes presented in Figure 1a. This enables the controlled optimization of the growth parameters within the selectivity window to achieve the desired morphologies, faceting, and highest crystal quality of the SAG structures. Moreover, we speculate that the combination of selectivity maps of binary compounds (e.g., InAs and GaAs) potentially can be extended to ternary alloys (e.g., $\operatorname{In}_{x} \mathrm{Ga}_{1-x} \mathrm{As}$ ) by isolating the intersecting areas on the maps where the growth proceeds selectively for both binary compounds composing the ternary alloy in question.

To demonstrate a wide applicability of developed technique, we have applied obtained knowledge to other material combinations and substrate orientations, which include growth of InAs, GaAs and InAs/GaAs SAG NWs on GaAs, InAs, and InP substrates of (001) and (111)B orientations with $\mathrm{SiO}_{x} / \mathrm{SiN}_{x}$ masks (see Supporting Information SD.1). Additionally, we have been able to apply the developed selectivity mapping technique to study the selectivity aspects of InSb SAG on various substrates. For example, we were able to successfully demonstrate homoepitaxial InSb SAG on $\operatorname{InSb}(001)$ and $\mathrm{InSb}(111) \mathrm{B}$ substrates, which suggests that our methodology can be extended beyond the III-As system (cf. Supporting Information SD.2). However, we have discovered that the growth and especially nucleation mechanisms differ for InSb in comparison to III-As SAG, which goes beyond the scope of the current work.

The case of InAs SAG on $\operatorname{InP}(111) \mathrm{B}$ substrate with $\mathrm{SiO}_{x}$ mask surface is studied in detail below because of its smaller lattice mismatch compared to GaAs substrate and of a 3-fold rotational surface symmetry [in contrast to a 2 -fold symmetry of the (001) surface], allowing the fabrication of structurally coherent nanostructure networks of identically faceted nanowires as desired for quantum computing applications. Refer to Supporting Information SD for a comparison of in-plane NW morphology on (001) and (111)B substrates supporting this choice.

An overview of the mask as well as of InAs single NW, junction, and network morphologies when grown on patterned $\operatorname{InP}(111) \mathrm{B}$ substrate is shown in Figure 2. For this, a prepared 2 in. wafer is loaded into MBE and growth of InAs proceeds with growth parameters chosen to be within the selectivity window outlined in Figure 1d1,d2. This way the in-plane shape of the NWs is guided by the mask pattern. Out-of-plane morphology of the NWs is governed by surface free energy minimization criteria and growth kinetics. For nanocircuit applications, precise control over both in-plane and out-of-plane shape must be achieved. This challenge can be separated into two parts: morphology of individual NWs and of their junctions. For individual NWs, their cross-section faceting strongly depends on the in-plane orientation of the InAs NWs in relation to the substrate orientation with uniform faceting obtained only in two high-symmetry direction families, $\langle 1 \overline{1} 0\rangle$ and $\langle 11 \overline{2}\rangle$ (Figure $2 \mathrm{~b})$. This is driven by facet surface energy minimization during the SAG, which enhances the facet growth along $\langle 1 \overline{1} 0\rangle$ and $<11 \overline{2}>$ directions as they correspond to local minima of the surface energy on the Wulff diagram for $\operatorname{InAs}(111) \mathrm{B} .^{54,55}$ Similar behavior was previously reported for GaAs(111)B inplane SAG structures. ${ }^{56}$ For NW junctions, their shape strongly depends on the relative orientation of joining NWs, with symmetrical junctions obtained only when the same NW direction family is used (Figure 2c). Note that $\langle 11 \overline{2}>$ are polar directions, and therefore, their junctions have different morphology when composed of $<11 \overline{2}>\mathrm{B}$ - versus $<11 \overline{2}>\mathrm{A}$ oriented NWs (Figure 2c, middle and bottom), with the latter one being more uniform in shape. All these aspects must be considered when designing the devices based on InAs NW networks (Figure 2d). Note that brightness and contrast variation in reported SEM images of NW networks originate from the morphology of the networks rather than from variation in their crystal or electrical properties, as no evidence to suggest otherwise was found by both structural and electrical characterizations presented further in the text.

Structural properties of uniformly faceted NWs oriented along high-symmetry $\langle 11 \overline{2}\rangle$ and $\langle 1 \overline{1} 0\rangle$ crystal directions (Figure 3a, left) were studied by scanning transmission electron microscopy (STEM) in high-angle annular dark-field (HAADF) mode. A thin $\mathrm{AlO}_{x}$ protective layer (grown by atomic layer deposition) was used for protection during the focused ion beam (FIB) preparation of the TEM lamellas, according to the method described in the Supporting Information $\mathrm{SH}^{57}$ The cross-sectional HAADF-STEM of $<11 \overline{2}>$-oriented NWs reveal their symmetrical shape (Figure $3 \mathrm{~b} 1$ ) with vertically oriented $\{1 \overline{1} 0\}$ side-walls, small inclusions of $\{201\}$ facets at the top corners, and a flat (111)B top facet. Shape symmetry agrees well with reflection symmetry of nonpolar $\{1 \overline{1} 0\}$ crystal side facets. No significant overgrowth on the mask is evident; the nanowire width is precisely defined by the opening area. White spots in the HAADF-STEM image are attributed to the re-evaporation of In during TEM lamella preparation. Atomic-resolution HAADFSTEM imaging shown in Figure $3 \mathrm{~b} 2$ reveals that InAs grows epitaxially on top of the InP substrate. Strain is mostly relaxed by the creation of a set of misfit dislocations at the interface between the two materials, as revealed in the rotational map obtained on the $(20 \overline{2})$ planes (Figure $3 \mathrm{~b} 3)$. Other than that, no defects have been found in the central and top part of the NW cross-section in agreement with reports on similar in-plane elongated nanostructures. ${ }^{24,58}$ However, this aspect was not systematically evaluated by TEM in this work due to a limited number of analyzed TEM lamellas and, therefore, does not 
exclude the possibility that extended defects are present in such wires.

The cross-section of $\langle 1 \overline{1} 0>$-oriented NW reveals its asymmetrical shape (Figure $3 \mathrm{c} 1$ ) with clear overgrowth to one side of the mask opening. This shape difference compared with $\langle 11 \overline{2}>-$ oriented NWs is explained by the absence of reflection symmetry of polar $<11 \overline{2}>$ directions (see Supporting Information SE.1). Atomic resolution STEM imaging shown in Figure $3 \mathrm{c} 2$ reveals an epitaxial relation of the $\operatorname{InAs}(111) \mathrm{B}$ SAG on $\operatorname{InP}(111) B$ substrates. Geometric phase analysis (GPA) of the InAs-InP interface reveals the presence of misfit dislocations similarly to the $\langle 11 \overline{2}>$-oriented NWs (Figure $3 c 3$ ), as expected. The NW cross-section characterization by atomic-resolution HAADF-STEM reveals its pure zincblende structure, being completely defect-free in the center and on the left side. However, some twin boundaries appear in the $(1 \overline{11})$ planes at the right side of the nanowire cross-section where the lateral overgrowth starts (Figure 3c4). We speculate that the formation of the twin is linked to the bond orientation of the As and In atoms at the $\mathrm{NW}$-mask interface. Indeed, it was reported that the mask step (from the sidewall to top surface) promotes bond distortion resulting in the anticlockwise rotation of the III-V crystal and $(1 \overline{11})$ twin-plane formation. ${ }^{59}$

Out of two inspected crystallographic directions, the $<11 \overline{2}>$ oriented NWs are chosen for further structural investigation because of their symmetric faceting and minimal overgrowth on the mask, both characteristics being favorable for high-quality NW networks formation. A longitudinal cross-section TEM lamella is prepared to investigate defect propagation along the NW. Some occasional stacking faults along $\{111\}$ planes are found in the structure (red arrows in Figure $3 \mathrm{~d} 2-\mathrm{d} 4$ ), which correspond to Frank partial dislocations. ${ }^{60}$ In the case of stacking faults that are not parallel to the substrate, they appear after several nanometers above the interface with the substrate and propagate until the top of the NW. Upon reaching the top facet of the NW, they create a step feature as depicted in Figure $3 \mathrm{~d} 3$. Occasional in-plane oriented stacking faults are also present, as shown in Figure 3d4. Despite that, most of the NW volume appears defect-free and exhibits zincblende structure with no wurtzite segments found in any of inspected TEM lamellas. We emphasize that the grown nanostructures show a perfect epitaxial relationship between the $\operatorname{InP}(111) \mathrm{B}$ substrate and the InAs grown NWs, while preserving the (111)B polarity of the substrate, as shown by the atomic resolution HAADF STEM analyses in Figure 3, as expected. ${ }^{61}$

Triple junctions consisting of $<11 \overline{2}>A$ and $<11 \overline{2}>B$ NWs (depicted in Figure $2 \mathrm{~b}$ top and bottom) are also examined by TEM; however, no significant difference compared to single $<11 \overline{2}>$-oriented NWs is found in the examined samples (see Supporting Information SG).

All TEM lamellas are also inspected for chemical composition uniformity via electron energy loss spectroscopy (EELS) elemental composition mapping. The chemical analyses reveal no detectable diffusion of the elements at the interface (see Supporting Information SF). In general, the InAs NW crystal quality is high, as is as the chemical purity. This agrees with recent studies by Krizek et al. ${ }^{21}$ and Friedl et al. ${ }^{24}$

Based on the results presented above, we choose the $\langle 11 \overline{2}\rangle$ NW family to construct NW networks for quantum transport characterization. Their symmetrical shape ensures structural coherence of InAs NW networks. Moreover, absence of overgrowth of $\langle 11 \overline{2}\rangle$-oriented wires allows accurate control over their dimensions by relatively simple means: height by adjusting the growth time, width by lithographically defining the width of the mask opening, and further adjustments by tuning the growth conditions to favor or hinder lateral growth. ${ }^{20}$

To demonstrate the potential of obtained InAs SAG networks for quantum transport applications, the Aharonov-Bohm effect is studied in differently sized loop-shaped NW networks at a temperature of $20 \mathrm{mK}$ (device fabrication and measurements details are described in the Supporting Information). An example of such a nanostructure with a circumference of about $3.9 \mu \mathrm{m}$ is shown in Figure 4a. Due to the finite width of the NW of about $190 \mathrm{~nm}$, the trajectories inside these loops can vary between the inner circumference of about $3.0 \mu \mathrm{m}$ and the outer circumference of about $4.6 \mu \mathrm{m}$. On average, the enclosed loop area corresponds to $0.9 \mu \mathrm{m}^{2}$. Also, loops with a circumference of about $13.4 \mu \mathrm{m}$ are investigated, where the enclosed area is an order of magnitude larger. As a common feature in mesoscopic devices, slowly varying universal conductance fluctuations are superimposed on the Aharonov-Bohm oscillations. ${ }^{18}$ This magnetoconductance variation is removed by fitting and subtracting a background in the form of a second-order polynomial. We find that the fast Fourier transform (FFT) of the conductance oscillations exhibits frequency contributions only within the shaded frequency interval in Figure $4 b, c$ (bottom panels), which corresponds to the possible values of the area enclosed by the loop trajectories inside the device. The dominating frequency corresponds to a period of $4.98 \mathrm{mT}$ for the smaller loop (cf. Figure $4 \mathrm{~b}$ ) and $0.45 \mathrm{mT}$ for the large loop (cf. Figure $4 \mathrm{~d}$ ), in agreement with the expected periods estimated from the loop area $A$ via $h / e A$. The small loop exhibits oscillation amplitudes on the order of $\Delta G=0.04 \times\left(2 e^{2} /\right.$ $h)$, while the large one yields a smaller value of $\Delta G=0.01 \times$ $\left(2 e^{2} / h\right)$, as expected. Our measurements demonstrate continuous conductance and phase-coherent transport in all sections (including the junctions) of extraordinarily large loop structures of dimensions relevant for interferometric readout of topological qubits. ${ }^{14,40}$ This, and the fact that epitaxial $\mathrm{Al}$ can be fabricated on studied InAs NWs (see Supporting Information SJ), suggest that the InAs SAG structures can serve as an excellent platform for future quantum transport experiments and topological braiding operations.

In conclusion, we have shown how to reliably obtain selectivity maps for SAG of III-V materials. The upper and lower bounds for impinging group III and V fluxes defining the selective growth parameter window were mapped for InAs and GaAs SAG cases. This potentially enables controlled material quality optimization within the full selective growth window. Experimental results are explained in terms of difference in desorption of group III and V elements from the mask versus the III-V surface. We identified that the desorption of group III element is the key mechanism governing the selectivity during SAG of III-V nanostructures. These results can generally be translated to other III-V materials and substrates and used to establish a parameter space where the selective regime can be achieved easily. Following this, advanced InAs/GaAs NW networks with unprecedent network complexity and shape control were realized on various substrates. Morphology, structural, and transport properties of in-plane InAs NW networks on semi-insulating $\operatorname{InP}(111) B$ substrates were studied in detail. Zincblende InAs NWs exhibit an epitaxial relation to $\operatorname{InP}(111) B$ substrates, with strain being efficiently relaxed following the creation of an array of misfit dislocations at the InAs-InP interface. Networks composed of nanowires oriented along $\langle 11 \overline{2}>$ orientations exhibit symmetrical uniform faceting, 

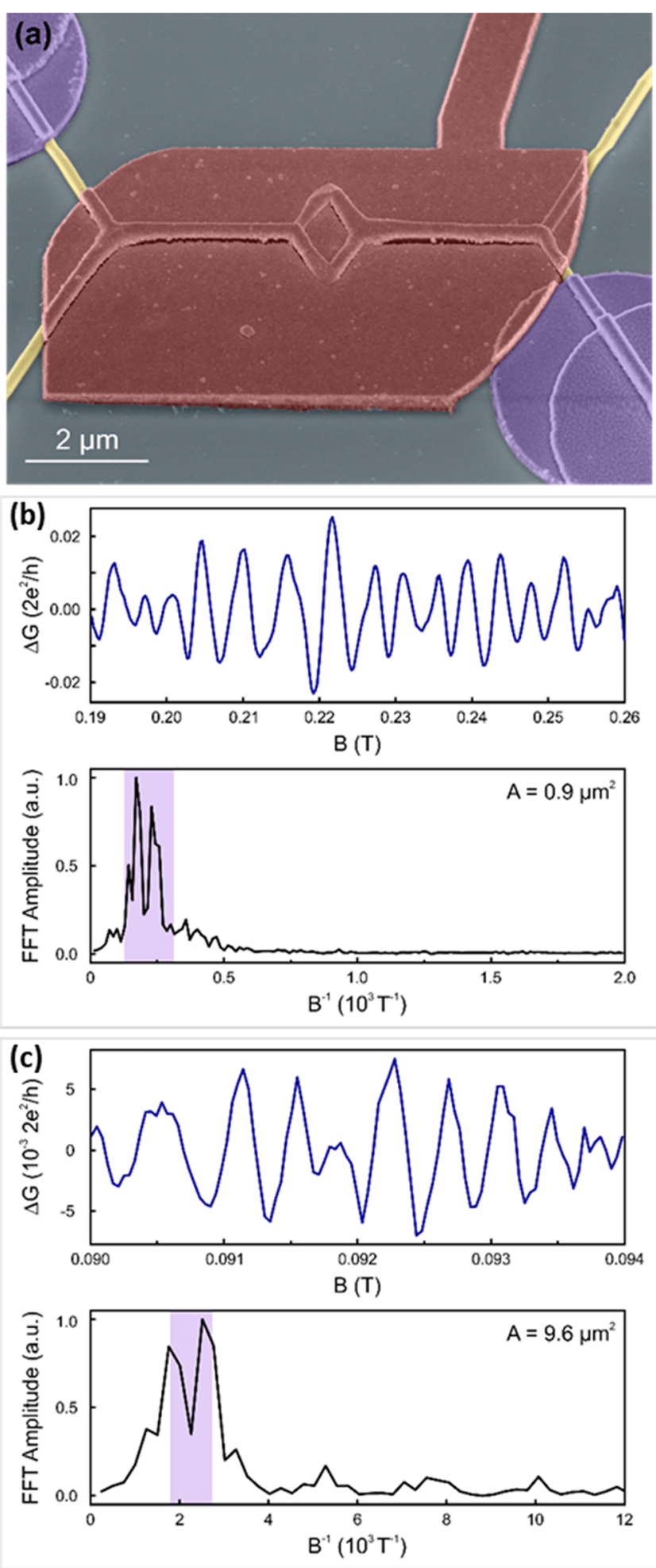

Figure 4. Aharonov-Bohm effect in InAs loop structures. (a) Scanning electron micrograph of an InAs SAG Aharonov-Bohm ring (yellow) contacted by $\mathrm{Ti} / \mathrm{Au}$ leads (purple) and covered with $\mathrm{SiN}_{x}$ dielectric and a top-gate electrode (red). The loop area (A) corresponds to $0.9 \mu \mathrm{m}^{2}$. (b) Periodic oscillations in magnetoconductance $\Delta G$ measured for the device depicted in panel a. A slowly varying background has been subtracted. In the bottom panel, the FFT spectrum of $\Delta G$ is presented, where the shaded region indicates the frequency range that is expected to contribute to the magnetoconductance due to the device dimensions. (c) Magnetoconductance oscillations measured in a much-larger loop structure with $A=9.6 \mu \mathrm{m}^{2}$. nearly defect-free structure, and an absence of overgrowth, which makes them preferable over other orientations for constructing structurally coherent networks. Obtained NW networks demonstrate phase-coherent electrical transport via the Aharonov-Bohm effect observed in differently sized loopshaped nanostructures. Phase coherence is maintained across devices with a circumference of more than $10 \mu \mathrm{m}$. This demonstrates the viability of the selectively grown NW networks as a platform for measurement-based quantum gates for topological quantum computing.

\section{ASSOCIATED CONTENT}

\section{Supporting Information}

The Supporting Information is available free of charge on the ACS Publications website at DOI: 10.1021/acs.nanolett.8b03733.

Additional details and figures on flux and substrate temperature calibration methods, mask preparation, selectivity mapping methodology, demonstration of networks grown on various substrates, demonstration of InSb SAG NW networks on InSb substrates, the morphology of differently oriented InAs NWs on both $<001>$ and $<111>$ B-oriented substrates, chemical composition uniformity of InAs SAG NWs, structural quality of NW junctions, FIB lamella preparation details, device fabrication and measurements, and epitaxial $\mathrm{Al}$ on InAs SAG NWs (PDF)

\section{AUTHOR INFORMATION}

\section{Corresponding Authors}

*E-mail: P.Aseev@tudelft.nl.

*E-mail: Leo.Kouwenhoven@microsoft.com.

*E-mail: Philippe.Caroff@microsoft.com.

\section{ORCID}

Pavel Aseev: 0000-0003-0343-9302

Jordi Arbiol: 0000-0002-0695-1726

\section{Author Contributions}

P. Aseev conceived the idea for the research, performed the growth, growth kinetic studies, and morphological analysis, and wrote most of the manuscript. A. Fursina developed the maskpreparation procedure and provided patterned substrates for the growth. F. Boekhout, F. Krizek, and J. E. Sestoft significantly participated in the growth, analysis, some device processing and measurements. S. Heedt, G. Wang, F. Borsoi, and L. Binci fabricated devices and performed the electrical characterization and analysis. S. Heedt wrote the corresponding part of the manuscript. S. Martí-Sánchez, T. Swoboda, and J. Arbiol performed TEM sample preparation and related structural and compositional analyses as well as wrote the corresponding section of the manuscript. R. Koops and E. Uccelli participated in the growth discussion and assisted with the growth. P. Caroff, L. P. Kouwenhoven, and P. Krogstrup supervised the work and provided extensive comments to the manuscript. All authors commented on the work and provided valuable input throughout the project as well as approved the final version of the manuscript.

\section{Notes}

The authors declare no competing financial interest. 


\section{ACKNOWLEDGMENTS}

The project was supported by Microsoft Station Q (Delft). P. Aseev and P. Caroff gratefully acknowledges Amrita Singh for mask preparation for some of the samples via a wet etching variant of the process. S. Marti-Sánchez acknowledges funding from "Programa Internacional de Becas "la Caixa"-Severo Ochoa”. ICN2 members acknowledge funding from Generalitat de Catalunya 2017 SGR 327. ICN2 acknowledges support from the Severo Ochoa Programme (MINECO, grant no. SEV-20130295) and is funded by the CERCA Programme/Generalitat de Catalunya. Part of the present work has been performed in the framework of Universitat Autònoma de Barcelona Materials Science PhD program. Part of the HAADF-STEM microscopy was conducted in the Laboratorio de Microscopias Avanzadas at Instituto de Nanociencia de Aragon-Universidad de Zaragoza.

\section{REFERENCES}

(1) Kitaev, A. Yu. Fault-Tolerant Quantum Computation by Anyons. Ann. Phys. 2003, 303 (1), 2-30.

(2) Stern, A.; Lindner, N. H. Topological Quantum ComputationFrom Basic Concepts to First Experiments. Science 2013, 339 (6124), 1179-1184.

(3) Sarma, S. D.; Freedman, M.; Nayak, C. Majorana Zero Modes and Topological Quantum Computation. Npj Quantum Inf. 2015, 1 (1), 15001.

(4) Zhang, H.; Liu, C.-X.; Gazibegovic, S.; Xu, D.; Logan, J. A.; Wang, G.; van Loo, N.; Bommer, J. D. S.; de Moor, M. W. A.; Car, D. Quantized Majorana Conductance. Nature 2018, 556, 74-79.

(5) Sestoft, J. E.; Kanne, T.; Gejl, A. N.; von Soosten, M.; Yodh, J. S.; Sherman, D.; Tarasinski, B.; Wimmer, M.; Johnson, E.; Deng, M.; et al. Engineering Hybrid Epitaxial InAsSb/Al Nanowires for Stronger Topological Protection. Phys. Rev. Mater. 2018, 2 (4), No. 044202.

(6) Lutchyn, R. M.; Bakkers, E. P. A. M.; Kouwenhoven, L. P.; Krogstrup, P.; Marcus, C. M.; Oreg, Y. Majorana Zero Modes in Superconductor-Semiconductor Heterostructures. Nat. Rev. Mater. 2018, 3 (5), 52-68.

(7) Deng, M. T.; Vaitiekénas, S.; Hansen, E. B.; Danon, J.; Leijnse, M.; Flensberg, K.; Nygård, J.; Krogstrup, P.; Marcus, C. M. Majorana Bound State in a Coupled Quantum-Dot Hybrid-Nanowire System. Science 2016, 354 (6319), 1557-1562.

(8) Krogstrup, P.; Ziino, N. L. B.; Chang, W.; Albrecht, S. M.; Madsen, M. H.; Johnson, E.; Nygård, J.; Marcus, C. M.; Jespersen, T. S. Epitaxy of Semiconductor-Superconductor Nanowires. Nat. Mater. 2015, 14, 400-406.

(9) Mourik, V.; Zuo, K.; Frolov, S. M.; Plissard, S. R.; Bakkers, E. P. A. M.; Kouwenhoven, L. P. Signatures of Majorana Fermions in Hybrid Superconductor-Semiconductor Nanowire Devices. Science 2012, 336 (6084), 1003-1007.

(10) Das, A.; Ronen, Y.; Most, Y.; Oreg, Y.; Heiblum, M.; Shtrikman, H. Zero-Bias Peaks and Splitting in an Al-InAs Nanowire Topological Superconductor as a Signature of Majorana Fermions. Nat. Phys. 2012, $8,887-895$.

(11) Deng, M. T.; Yu, C. L.; Huang, G. Y.; Larsson, M.; Caroff, P.; Xu, H. Q. Anomalous Zero-Bias Conductance Peak in a Nb-InSb Nanowire-Nb Hybrid Device. Nano Lett. 2012, 12 (12), 6414-6419.

(12) Wagner, R. S.; Ellis, W. C. Vapor-Liquid-Solid Mechanism of Signle Crystal Growth. Appl. Phys. Lett. 1964, 4 (5), 89-90.

(13) Jacobsson, D.; Panciera, F.; Tersoff, J.; Reuter, M. C.; Lehmann, S.; Hofmann, S.; Dick, K. A.; Ross, F. M. Interface Dynamics and Crystal Phase Switching in GaAs Nanowires. Nature 2016, 531 (7594), 317-322.

(14) Plugge, S.; Rasmussen, A.; Egger, R.; Flensberg, K. Majorana Box Qubits. New J. Phys. 2017, 19 (1), No. 012001.

(15) Car, D.; Wang, J.; Verheijen, M. A.; Bakkers, E. P. A. M.; Plissard, S. R. Rationally Designed Single-Crystalline Nanowire Networks. Adv. Mater. 2014, 26 (28), 4875-4879.
(16) Heedt, S.; Vakulov, D.; Rieger, T.; Rosenbach, D.; Trellenkamp, S.; Grützmacher, D.; Lepsa, M. I.; SchäPers, T. Electronic Properties of Complex Self-Assembled InAs Nanowire Networks. Adv. Electron. Mater. 2016, 2 (6), 01500460.

(17) Krizek, F.; Kanne, T.; Razmadze, D.; Johnson, E.; Nygård, J.; Marcus, C. M.; Krogstrup, P. Growth of InAs Wurtzite Nanocrosses from Hexagonal and Cubic Basis. Nano Lett. 2017, 17 (10), 60906096.

(18) Gazibegovic, S.; Car, D.; Zhang, H.; Balk, S. C.; Logan, J. A.; de Moor, M. W. A.; Cassidy, M. C.; Schmits, R.; Xu, D.; Wang, G.; et al. Epitaxy of Advanced Nanowire Quantum Devices. Nature 2017, 548, 434-438.

(19) Czornomaz, L.; Uccelli, E.; Sousa, M.; Deshpande, V.; Djara, V.; Caimi, D.; Rossell, M. D.; Erni, R.; Fompeyrine, J. Confined Epitaxial Lateral Overgrowth (CELO): A Novel Concept for Scalable Integration of CMOS-Compatible InGaAs-on-Insulator MOSFETs on Large-Area Si Substrates. IEEE Symposium on VLSI Technology 2015, T172-T173.

(20) Ujiie, Y.; Nishinaga, T. Epitaxial Lateral Overgrowth of GaAs on a Si Substrate. Jpn. J. Appl. Phys. 1989, 28 (2), L337-L339.

(21) Krizek, F.; Sestoft, J. E.; Aseev, P.; Marti-Sanchez, S.; Vaitiekénas, S.; Casparis, L.; Khan, S. A.; Liu, Y.; Stankevič, T.; Whiticar, A. M. Field Effect Enhancement in Buffered Quantum Nanowire Networks. Phys. Rev. Mater. 2018, 2 (9), 093401.

(22) Lee, J. S.; Choi, S.; Pendharkar, M.; Pennachio, D. J.; Markman, B.; Rodwell, M. J. W.; Krogstrup, P.; Palmstrøm, C. J. Selective-Area Chemical Beam Epitaxy of In-Plane InAs One-Dimensional Channels Grown on $\operatorname{InP}(001)$ and $\operatorname{InP}(111) B$ Surfaces. 2018, ArXiv:1808.04563v1 Cond-Mat. arXiv.org e-Print archive, https://arxiv. org/abs/1808.04563 (accessed August 14, 2018).

(23) Vaitiekénas, S.; Whiticar, A. M.; Deng, M.-T.; Krizek, F.; Sestoft, J. E.; Palmstrøm, C. J.; Marti-Sanchez, S.; Arbiol, J.; Krogstrup, P.; Casparis, L. Selective Area Grown Semiconductor-Superconductor Hybrids: A Basis for Topological Networks. Phys. Rev. Lett. 2018, 121, 147701.

(24) Friedl, M.; Cerveny, K.; Weigele, P.; Tütüncüoglu, G.; MartíSánchez, S.; Huang, C.; Patlatiuk, T.; Potts, H.; Sun, Z.; Hill, M. O.; et al. Template-Assisted Scalable Nanowire Networks. Nano Lett. 2018, 18 (4), 2666-2671.

(25) Gooth, J.; Borg, M.; Schmid, H.; Schaller, V.; Wirths, S.; Moselund, K.; Luisier, M.; Karg, S.; Riel, H. Ballistic One-Dimensional InAs Nanowire Cross-Junction Interconnects. Nano Lett. 2017, 17 (4), 2596-2602.

(26) Schmid, H.; Borg, M.; Moselund, K.; Gignac, L.; Breslin, C. M.; Bruley, J.; Cutaia, D.; Riel, H. Template-Assisted Selective Epitaxy of III-V Nanoscale Devices for Co-Planar Heterogeneous Integration with Si. Appl. Phys. Lett. 2015, 106 (23), 233101.

(27) Kim, J. D.; Chen, X.; Coleman, J. J. Selective Area Masked Growth (Nano to Micro). In Handbook of Crystal Growth; Elsevier: Amsterdam, The Netherlands, 2015; pp 441-481.

(28) Cho, A. Y. Recent Developments in Molecular Beam Epitaxy (MBE). J. Vac. Sci. Technol. 1979, 16 (2), 275-284.

(29) Okamoto, A.; Ohata, K. Selective Epitaxial Growth of Gallium Arsenide by Molecular Beam Epitaxy. Appl. Phys. Lett. 1987, 51 (19), $1512-1514$

(30) Okamoto, A.; Ohata, K. Selective Epitaxial Growth of InAs on GaAs by Molecular Beam Epitaxy. Jpn. J. Appl. Phys. 1987, 26 (2), L1174-L1176.

(31) Cho, A. Y.; Ballamy, W. C. GaAs Planar Technology by Molecular Beam Epitaxy (MBE). J. Appl. Phys. 1975, 46 (2), 783-785.

(32) Okamoto, A.; Ohata, K. Growth Condition Dependence for GaAs Selective Epitaxial Growth by Molecular Beam Epitaxy. J. Electron. Mater. 1989, 18 (2), 111-115.

(33) Okamoto, A.; Ohata, K. Substrate Temperature Lowering in GaAs Selective Epitaxial Growth by Molecular-beam Epitaxy. J. Appl. Phys. 1989, 66 (7), 3413-3415.

(34) Lee, S. C.; Malloy, K. J.; Dawson, L. R.; Brueck, S. R. J. Selective Growth and Associated Faceting and Lateral Overgrowth of GaAs on a 
Nanoscale Limited Area Bounded by a SiO2Mask in Molecular Beam Epitaxy. J. Appl. Phys. 2002, 92 (11), 6567-6571.

(35) Heiß, M.; Riedlberger, E.; Spirkoska, D.; Bichler, M.; Abstreiter, G.; Morral, A. F. i. Growth Mechanisms and Optical Properties of GaAs-Based Semiconductor Microstructures by Selective Area Epitaxy. J. Cryst. Growth 2008, 310 (6), 1049-1056.

(36) Allegretti, F.; Inoue, M.; Nishinaga, T. In-Situ Observation of GaAs Selective Epitaxy on GaAs (111)B Substrates. J. Cryst. Growth 1995, 146 (1-4), 354-358.

(37) Yokoyama, S.; Oogi, J.; Yui, D.; Kawabe, M. Low-Temperature Selective Growth of GaAs by Alternately Supplying Molecular Beam Epitaxy. J. Cryst. Growth 1989, 95 (1-4), 32-34.

(38) Tutuncuoglu, G.; de la Mata, M.; Deiana, D.; Potts, H.; Matteini, F.; Arbiol, J.; Fontcuberta i Morral, A. Towards Defect-Free 1-D GaAs/ AlGaAs Heterostructures Based on GaAs Nanomembranes. Nanoscale 2015, 7 (46), 19453-19460.

(39) Fahed, M.; Desplanque, L.; Coinon, C.; Troadec, D.; Wallart, X. Impact of $\mathrm{P} /$ In Flux Ratio and Epilayer Thickness on Faceting for Nanoscale Selective Area Growth of InP by Molecular Beam Epitaxy. Nanotechnology 2015, 26 (29), 295301.

(40) Vijay, S.; Fu, L. Teleportation-Based Quantum Information Processing with Majorana Zero Modes. Phys. Rev. B: Condens. Matter Mater. Phys. 2016, 94 (23), 235446 DOI: 10.1103/PhysRevB.94.235446.

(41) Foxon, C. T.; Joyce, B. A. Surface Processes Controlling the Growth of GaxIn1-xAs and GaxIn1-xP Alloy Films by MBE. J. Cryst. Growth 1978, 44 (1), 75-83.

(42) Sugaya, T.; Okada, Y.; Kawabe, M. Selective Growth of GaAs by Molecular Beam Epitaxy. Jpn. J. Appl. Phys. 1992, 31 (2), L713-L716.

(43) Däweritz, L.; Hey, R. Reconstruction and Defect Structure of Vicinal GaAs(001) and AlxGa1-xAs(001) Surfaces during MBE Growth. Surf. Sci. 1990, 236 (1-2), 15-22.

(44) Neave, J. H.; Joyce, B. A.; Dobson, P. J.; Norton, N. Dynamics of Film Growth of GaAs by MBE from Rheed Observations. Appl. Phys. A: Solids Surf. 1983, 31 (1), 1-8.

(45) Van Hove, J. M.; Cohen, P. I. Mass-action Control of AlGaAs and GaAs Growth in Molecular Beam Epitaxy. Appl. Phys. Lett. 1985, 47 (7), 726-728.

(46) Loitsch, B.; Rudolph, D.; Morkötter, S.; Döblinger, M.; Grimaldi, G.; Hanschke, L.; Matich, S.; Parzinger, E.; Wurstbauer, U.; Abstreiter, G.; et al. Tunable Quantum Confinement in Ultrathin, Optically Active Semiconductor Nanowires Via Reverse-Reaction Growth. Adv. Mater. 2015, 27 (13), 2195-2202.

(47) Lüth, H. Surfaces and Interfaces of Solids; Springer: Berlin, Germany, 1993.

(48) Plissard, S.; Dick, K. A.; Larrieu, G.; Godey, S.; Addad, A.; Wallart, X.; Caroff, P. Gold-Free Growth of GaAs Nanowires on Silicon: Arrays and Polytypism. Nanotechnology 2010, 21 (38), 385602.

(49) Colombo, C.; Spirkoska, D.; Frimmer, M.; Abstreiter, G.; Fontcuberta i Morral, A. Ga-Assisted Catalyst-Free Growth Mechanism of GaAs Nanowires by Molecular Beam Epitaxy. Phys. Rev. B: Condens. Matter Mater. Phys. 2008, 77 (15), 155326 DOI: 10.1103/PhysRevB.77.155326.

(50) Allegretti, F. E.; Nishinaga, T. Periodic Supply Epitaxy: A New Approach for the Selective Area Growth of GaAs by Molecular Beam Epitaxy. J. Cryst. Growth 1995, 156 (1-2), 1-10.

(51) Horikoshi, Y. Advanced Epitaxial Growth Techniques: Atomic Layer Epitaxy and Migration-Enhanced Epitaxy. J. Cryst. Growth 1999, 201-202, 150-158.

(52) Bacchin, G.; Nishinaga, T. A Detailed Comparison of the Degree of Selectivity, Morphology and Growth Mechanisms between PSE/ MBE and Conventional MBE. J. Cryst. Growth 1999, 198-199, 11301135.

(53) Bachrach, R. Z. On the Possibility of MBE Growth Interface Modification by Hydrogen. J. Vac. Sci. Technol., B: Microelectron. Process. Phenom. 1983, 1 (2), 142-145.

(54) Moll, N.; Kley, A.; Pehlke, E.; Scheffler, M. GaAs Equilibrium Crystal Shape from First Principles. Phys. Rev. B: Condens. Matter Mater. Phys. 1996, 54 (12), 8844-8855.
(55) Krogstrup, P.; Jørgensen, H. I.; Johnson, E.; Madsen, M. H.; Sørensen, C. B.; Morral, A. F. i; Aagesen, M.; Nygård, J.; Glas, F. Advances in the Theory of III-V Nanowire Growth Dynamics. J. Phys. D: Appl. Phys. 2013, 46 (31), 313001.

(56) Molecular Beam Epitaxy: From Research to Mass Production; Henini, M., Ed.; Elsevier: Amsterdam, The Netherlands, 2013.

(57) Giannuzzi, L. A.; Drown, J. L.; Brown, S. R.; Irwin, R. B.; Stevie, F. A. Focused Ion Beam Milling and Micromanipulation Lift-Out for Site Specific Cross-Section Tem Specimen Preparation. MRS Online Proc. Libr. 1997, 480, 19 DOI: 10.1557/PROC-480-19.

(58) Chi, C.-Y.; Chang, C.-C.; Hu, S.; Yeh, T.-W.; Cronin, S. B.; Dapkus, P. D. Twin-Free GaAs Nanosheets by Selective Area Growth: Implications for Defect-Free Nanostructures. Nano Lett. 2013, 13 (6), 2506-2515.

(59) Renard, C.; Cherkasin, N.; Jaffre, A.; Vincent, L.; Michel, A.; Molière, T.; Hamouche, R.; Yam, V.; Alvarez, J.; Fossard, F.; et al. Dislocation and Antiphase Domain Free Microscale GaAs Crystals Grown on $\mathrm{SiO}_{2}$ from (001) Si Nano-Areas. Appl. Phys. Lett. 2013, 102 (19), 191915.

(60) Arroyo Rojas Dasilva, Y.; Kozak, R.; Erni, R.; Rossell, M. D. Structural Defects in Cubic Semiconductors Characterized by Aberration-Corrected Scanning Transmission Electron Microscopy. Ultramicroscopy 2017, 176, 11-22.

(61) de la Mata, M.; Magen, C.; Gazquez, J.; Utama, M. I. B.; Heiss, M.; Lopatin, S.; Furtmayr, F.; Fernández-Rojas, C. J.; Peng, B.; Morante, J. R; et al. Polarity Assignment in $\mathrm{ZnTe}, \mathrm{GaAs}, \mathrm{ZnO}$, and GaN-AlN Nanowires from Direct Dumbbell Analysis. Nano Lett. 2012, 12 (5), 2579-2586. 